\title{
Predictive Current Control Strategy for a Multi-modular Matrix Converter
}

\author{
Diego Rojas, Marco Rivera, Patrick Wheeler
}

\begin{abstract}
Matrix converter technology have so far been mainly limited to low power applications. Multi-level voltage source converters are often used for high power applications. In order to combine the good characteristics of both topologies this paper presents the principle of operation of multi-modular matrix converters. Among all the proposed control techniques, predictive control has become a viable and highly versatile option due to its ability to cope with different operating requirements. Therefore this paper also proposes a predictive control technique with a fixed switching frequency applied to the multi-modular matrix converter as well as a comparison of the performance between the proposed control and a variable switching frequency counterpart, showing the advantages and disadvantages between the two strategies.
\end{abstract}

Keywords-Current control, fixed frequency, multi-modular converter, power converter, single phase converter, predictive control.

\section{INTRODUCCIÓN}

$\mathbf{E}$ L convertidor de potencia forma una parte importante para el manejo de la energía eléctrica, proporcionando la interconexión entre la generación y distribución. Los convertidores de potencia más populares incluyen elementos almacenadores de energía, los cuáles introducen posibilidades a fallas, como también mayor peso y tamaño. El convertidor matricial es una topología de convertidor de potencia que no tienen almacenadores de energía, por eso, son una alternativa flexible y eficiente para el manejo de la energía [1].

La topología del convertidor matricial (MC) ha llamado la atención para aplicaciones de alta potencia. Sin embargo, las limitaciones de voltaje de los semiconductores utilizados para la conmutación de los switches en el convertidor matricial tradicional $(3 \times 3)$ no son una opción viable. Para ello, se utiliza la topología de los Convertidores Matriciales Multimodulares (MMMC), formadas por la conexión en cascadas y en serie de tres convertidores matriciales monofásicas [2], como se observa en la Fig. 1.

El control predictivo basado en modelo (MPC) se introdujo a finales de los setenta y en las últimas décadas se ha introducido para el control de los convertidores de potencia

\footnotetext{
D. Rojas. Estudiante del Doctorado en Sistemas de Ingeniería, Facultad de Ingeniería, Universidad de Talca, Curicó, Chile (e-mail: diego.rojaso@utalca.cl)

M. Rivera. Director del Laboratorio de Conversión de Energías y Electrónica de Potencia, Universidad de Talca, Curicó, Chile (e-mail: marcoriv@utalca.cl)

P. Wheeler. Power Electronics, Machines and Control Group, The University of Nottingham, Nottingham, UK (e-mail: Pat.Wheeler@nottingham.ac.uk)
}

978-0-7381-3333-1/21\$31.00 C2021 IEEE y diversas aplicaciones. Mostrando en varios casos, un mejor rendimiento que las técnicas de control clásicas PWM [3].

El control predictivo clásico, calcula la predicción del comportamiento del sistema, minimiza la función de costo para cierto estado de conmutación y se aplica el vector de voltaje correspondiente a dicho estado. El MPC clásico produce una frecuencia de conmutación variable lo cual podría ocasionar eventualmente resonancias en los filtros del convertidor de potencia, afectando el desempeño del sistema [4].

En este artículo, se aplicará la técnica de control predictivo a frecuencia de conmutación fija, que se basa en seleccionar dos vectores óptimos para su aplicación en ciclos de tiempo definidos por sus factores de ponderación en la función de costo, junto con los vectores cero, en cada instante de muestreo [5]. Varios trabajos han abordado esta problemática recientemente, en los cuales se han reportado mejoras en términos de distorsión armónica total (THD) para control de corriente, par mecánico, flujo electromagnético y control de potencia tanto activa como reactiva [6].

De esta forma, la principal contribución de este trabajo, se centra en un análisis teórico del rendimiento del control predictivo a frecuencia fija en comparación con el control predictivo a frecuencia variable, aplicados a un convertidor matricial multi-modular. Para validar el método propuesto, las simulaciones se llevan a cabo utilizando el software MATLAB/Simulink.

\section{TÉCNICA MPC APLICADO AL MMMC}

El MMMC, permite una distribución de potencia entre sus semiconductores con lo cual es posible controlar potencias mayores en base a semiconductores de menor potencia. Como es bien sabido, los convertidores multinivel pueden generar voltajes de salida con bajas distorsiones armónicas totales (THD) y reducir el estrés de voltaje para lograr una conversión de alta potencia [7].

\section{A. Topología del MC multi-modular}

La topología del convertidor matricial 3x1-modular se observa en la Fig. 1, su celda básica es un convertidor matricial monofásica (SPMC) [8], que consta de seis interruptores de potencia bidireccionales, y cada interruptor de potencia está compuesto por dos IGBT con pares de diodos antiparalelos conectados al emisor común. En la nomenclatura 3x1-modular, " 3 " significa que el convertidor tiene salida trifásica, y " 1 " significa que cada fase tiene solo un SPMC. Una aplicación del SPMC multi-modular, es el control para un motor trifásico como se observa en la Fig. 1 [9]. 


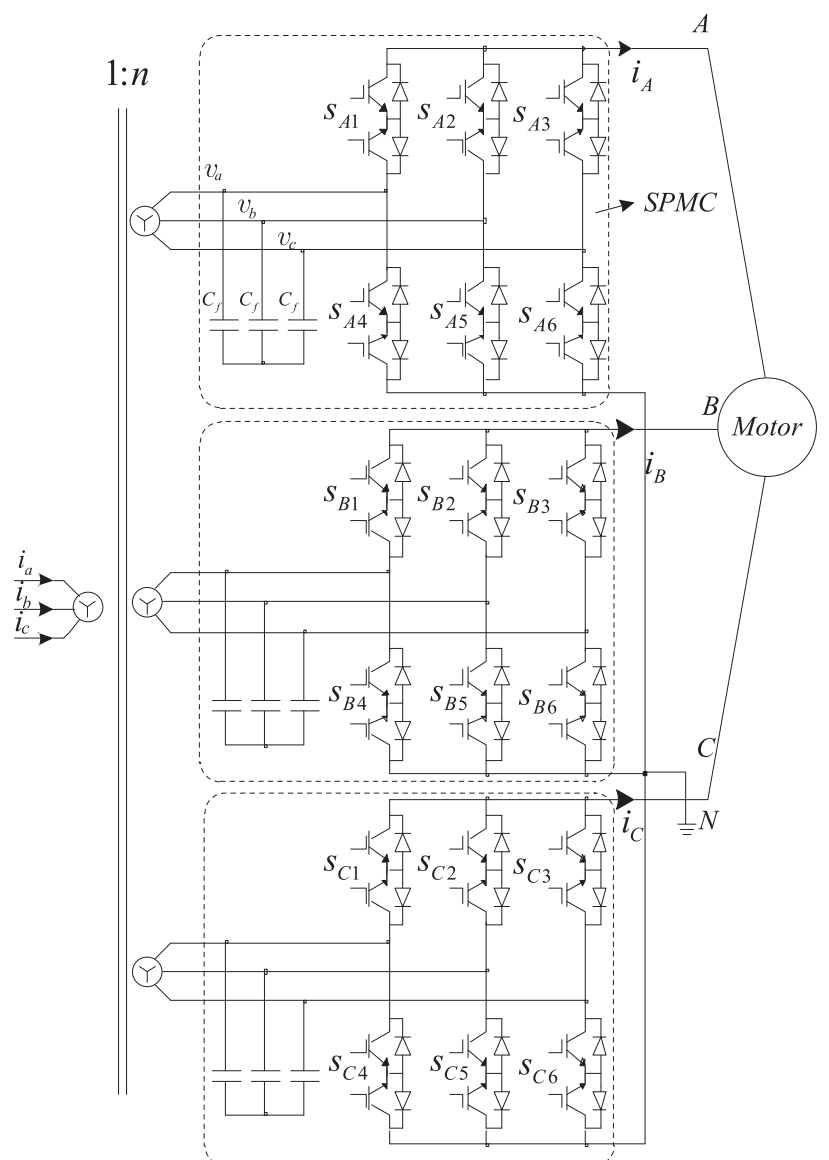

Fig. 1. Estructura de la topología $3 \times 1$-modular MC.

Para obtener una tensión más elevada y más niveles de tensión de salida, se deben conectar varias celdas SPMC en serie. Por ejemplo, un MC multi-modular con $N$ SPMC conectadas en serie en cada fase se ilustra en la Fig. 2, esta topología se denomina $3 \times N$-modular MCs.

\section{B. Modelo matemático del convertidor matricial monofásico (SPMC) $3 \times 1-$ modular}

De la Fig. 1, las tensiones de salida y corriente de entrada se puede escribir de la siguiente manera:

$$
\begin{aligned}
v_{x}=\left(S_{x 1}-S_{x 4}\right) & v_{a}+\left(S_{x 2}-S_{x 5}\right) v_{b}+\left(S_{x 3}-S_{x 6}\right) v_{c} \\
i_{a} & =\sum_{x \in(A, B, C)}\left(S_{x 1}-S_{x 4}\right) i_{x} \\
i_{b} & =\sum_{x \in(A, B, C)}\left(S_{x 2}-S_{x 5}\right) i_{x} \\
i_{c} & =\sum_{x \in(A, B, C)}\left(S_{x 3}-S_{x 6}\right) i_{x}
\end{aligned}
$$

Donde $v_{a}, v_{b}, v_{c}$ son los voltajes de entrada al SPMC multimodular, $S_{x y}$ denota el estado de cada interruptor bidireccional, $x \in(A, B, C), y \in(1,2,3,4,5,6)$.

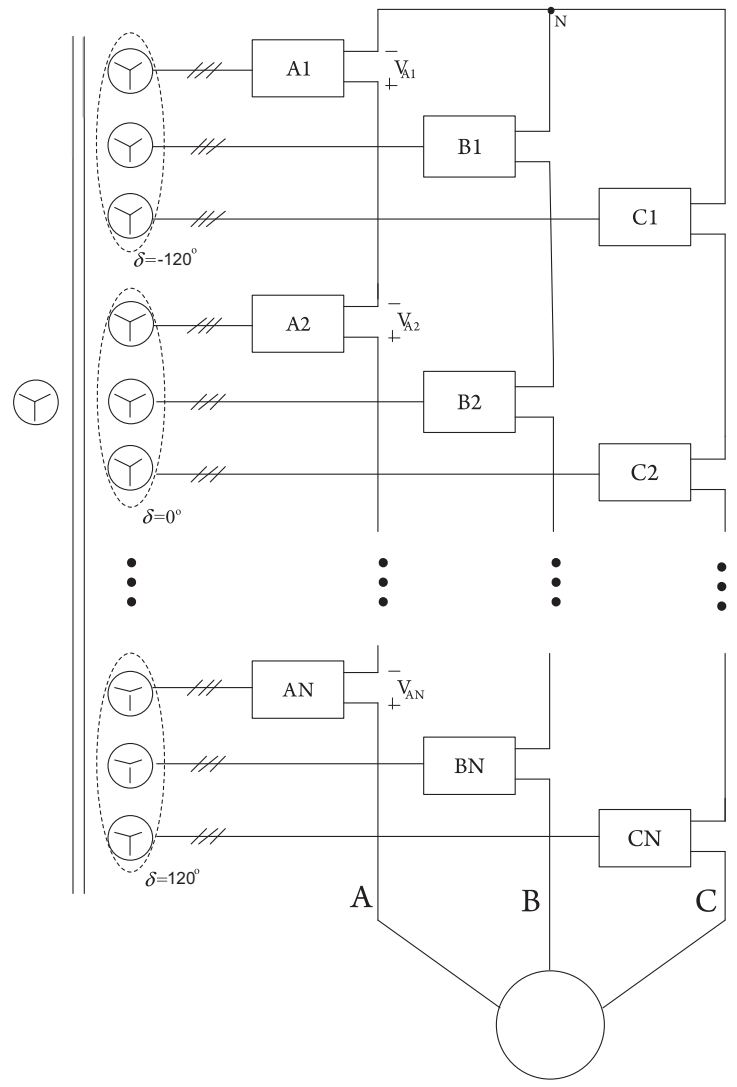

Fig. 2. Diagrama simplificado del $3 \times N$-modular MC.

\section{Esquema de control}

La Fig. 3 representa el esquema de control predictivo aplicado al SPMC multi-modular, en el cual se aprecia la interacción entre el MPC y el convertidor matricial multi-modular. Se aplicará de nuevo el control de corriente a la salida, para el efecto se usará una carga trifásica equilibrada resistiva-inductiva, la ecuación matemática para la predicción de la corriente futura [10] aplicada para cada fase de salida está dada por la ecuación (3).

$$
i_{x}(k+1)=\frac{T_{s}}{L} v_{x}(k)+\left(1-\frac{R T_{s}}{L}\right) i_{x}(k)
$$

donde:

$x \in(A, B, C):$ fases de salida del SPMC multi-modular $v_{x}(k)$ : tensión actual de salida del SPMC multi-modular $[V]$

$i_{x}(k)$ : corriente actual en la carga para cada fase de salida del SPMC multi-modular $[A]$

$i_{x}(k+1): \quad$ corriente predicha para cada fase de salida del SPMC multi-modular $[A]$

$T_{s}: \quad$ periodo de muestreo $[s]$

$L: \quad$ inductancia de las cargas $[H]$

$R$ : resistencia de las cargas $[\Omega]$

Las concatenaciones de las ecuaciones (1) al (3), con los estados válidos para cada SPMC a aplicar, generan el modelo de predicción de la corriente en la carga. 


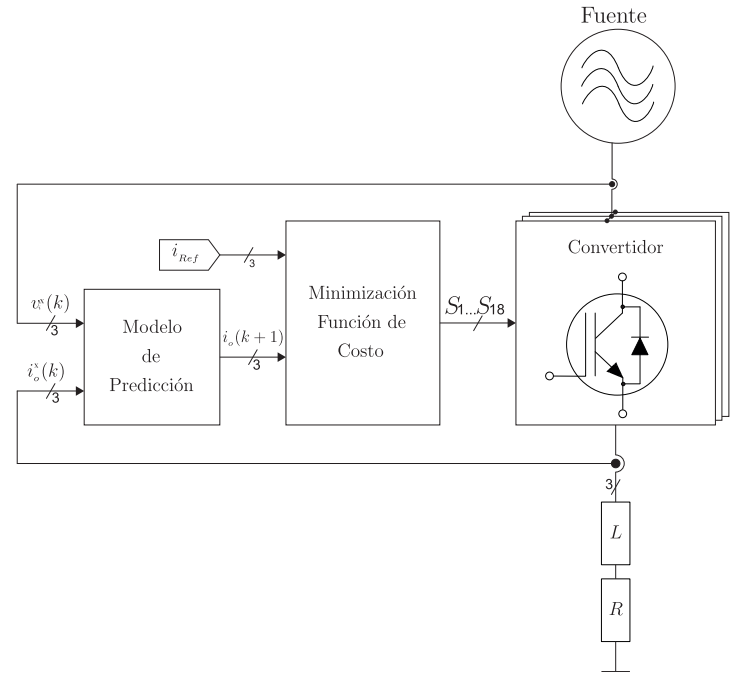

Fig. 3. Esquema de control MPC aplicado al MMMC.

\section{Función de costo}

La función de costo busca optimizar la resultante del sistema, con el fin de minimizar los costos que generan las acciones de control. En este caso se desea que la salida del convertidor siga a una referencia de corriente trifásica desfasadas $120^{\circ}$ con características periódicas como lo son la magnitud, la frecuencia y la forma. En consecuencia, lo esperado es que la salida sea idéntica a la referencia, por lo tanto, una función de costo que cumple con este requerimiento es el error entre la corriente de referencia y la corriente de carga para cada fase:

$$
g_{x}(k+1)=\left(i_{r e f_{x}}(k+1)-i_{x}(k+1)\right)^{2}
$$

donde: $x \in(A, B, C)$

\section{E. Técnica de control MPC a frecuencia de conmutación fija aplicado al MMMC}

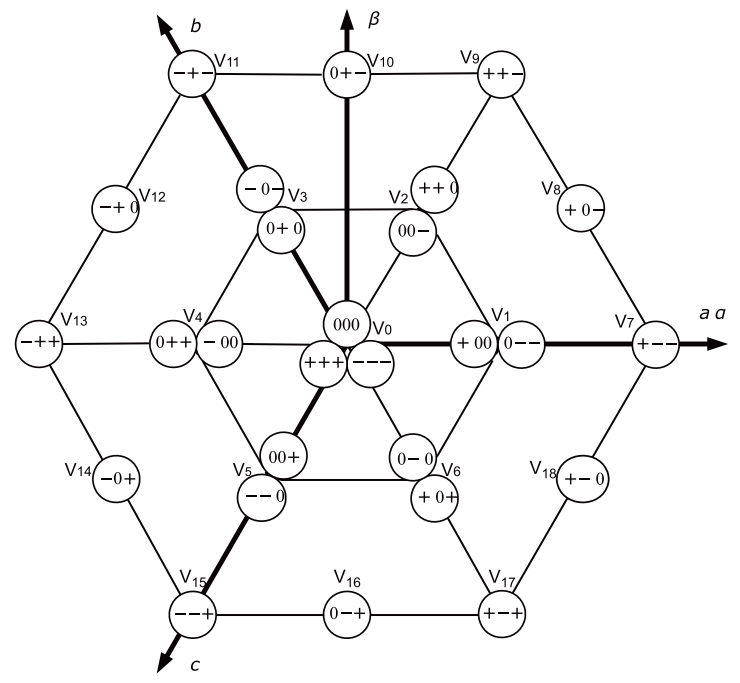

Fig. 4. Vectores de tensión válidos en el plano $\alpha-\beta$.
Es posible definir cada vector de tensión válidos para el MMMC en el plano $\alpha-\beta$ [11], como se muestra en la Fig. 4. El MPC a frecuencia de conmutación fija [12], se basa en seleccionar dos vectores óptimos $\left(v_{\text {opt } 1}, v_{\text {opt } 2}\right)$ adyacentes correspondiente al mismo sector para su aplicación en ciclos de tiempo definidos dependiendo de la función de costo, junto con los vectores cero $\left(v_{0}\right)$. Para aplicar la modulación, los ciclos de trabajo asociados a los vectores se calculan definiéndolos inversamente proporcional al valor de la función de costo, es decir, si la función de costo evaluada en un vector posee un valor elevado, el ciclo de trabajo es reducido, en consecuencia, los ciclos de trabajos para los vectores de voltaje se calculan resolviendo:

$$
d_{0}=\frac{\delta}{g_{0}}, d_{1}=\frac{\delta}{g_{1}}, d_{2}=\frac{\delta}{g_{2}}
$$

donde $\delta$ es la constante de proporcionalidad, $g_{1}$ y $g_{2}$ denotan las funciones de costo definida en la ecuación (4) evaluadas para los vectores óptimos $\left(v_{o p t 1}, v_{o p t 2}\right)$ y $g_{o}$ corresponde al vector cero $\left(v_{0}\right)$, el cual se evalúa en cada instante de muestreo. Además, se tiene que:

$$
d_{0}+d_{1}+d_{2}=1
$$

calculando la función de costo para cada uno de los vectores óptimos es posible determinar el valor de $\delta$ sustituyendo la ecuación (5) en la (6). Una vez calculado el valor de $\delta$ y teniendo en cuenta la ecuación (5), los ciclos de trabajo están dados entonces por:

$$
\begin{aligned}
& d_{0}=\frac{g_{1} g_{2}}{g_{0} g_{1}+g_{1} g_{2}+g_{0} g_{2}} \\
& d_{1}=\frac{g_{0} g_{2}}{g_{0} g_{1}+g_{1} g_{2}+g_{0} g_{2}} \\
& d_{2}=\frac{g_{0} g_{1}}{g_{0} g_{1}+g_{1} g_{2}+g_{0} g_{2}}
\end{aligned}
$$

Considerando estas expresiones, la nueva función de costo, que se evalúa en cada tiempo de muestreo, se define como:

$$
g=d_{1} g_{1}+d_{2} g_{2}
$$

La combinación de vectores que minimicen la ecuación (10) y el vector cero son seleccionados y aplicados al MMMC en el siguiente tiempo de muestreo. Luego de obtener los ciclos de trabajo y los dos vectores óptimos a ser aplicados, se definen los tiempos de aplicación de cada vector como sigue:

$$
\begin{aligned}
& T_{0}=T_{s} d_{0} \\
& T_{1}=T_{s} d_{1} \\
& T_{2}=T_{s} d_{2}
\end{aligned}
$$

donde $T_{s}$ es el tiempo de muestreo.

La predicción de la corriente de carga para cada fase se obtiene por la ecuación (3), los ciclos de trabajo de cada vector óptimo se calculan por las ecuaciones (7), (8) y (9) pero para cada SPMC, donde las funciones de costo $\left(g_{0}, g_{1}, g_{2}\right)$ se calculan para cada corriente de salida de cada fase, para luego aplicar la ecuación (10) en cada fase y en los diferentes tiempos de aplicación definidas en la ecuación (11) para cada SPMC. 


\section{RESUlTADOS DE SIMULACIÓN}

La simulación de los controladores expuestos sobre el MMMC se realizaron en el entorno de simulación Matlab/Simulink para un seguimiento de corriente. En la Tabla I se puede apreciar los distintos parámetros empleados en la simulación.

Tabla I

PARÁMETROS DE SIMULACIÓN

\begin{tabular}{c|c|c|c}
\hline Variable & Descripción & Valor & Unidad \\
\hline$v_{i}$ & Tensión de la fuente & 1620 & Vrms \\
$f_{i}$ & Frecuencia de la fuente & 50 & $H z$ \\
$T_{s}$ & Tiempo de muestreo & $10-25$ & $\mu s$ \\
$R$ & Resistencia de carga & 10 & $\Omega$ \\
$L$ & Inductancia de carga & 10 & $m H$ \\
$R_{f}$ & Resistencia del filtro & 0.5 & $\Omega$ \\
$L_{f}$ & Inductancia del filtro & 400 & $\mu H$ \\
$C_{f}$ & Capacitancia del filtro & 25 & $\mu F$ \\
$R_{d}$ & Resist. de amortiguamiento & 56 & $\Omega$ \\
$i_{r e f}$ & Corriente de referencia & 40 & $A$ \\
$f_{r e f}$ & Frecuencia de referencia & 50 & $H z$ \\
\hline
\end{tabular}

Se han realizado simulaciones de diferentes escenarios para mostrar la velocidad de respuesta y la exactitud de la técnica.

\section{A. Resultado en estado estable}

En la primera prueba de los controladores se evaluó su comportamiento bajo un estado estable. Es decir, se evaluó el seguimiento de una referencia de características cíclicas y continuas en el tiempo. Las Figs. 5 y $\mathbf{6}$ muestran los resultados de simulación en estado estacionario para la corriente de carga y tensión de carga, utilizando un tiempo de muestreo de $T_{s}=25[\mu s]$ y $T_{s}=10[\mu s]$, respectivamente, obtenidos al aplicar la técnica de control predictivo basado en modelo clásico al MMMC. Comparando ambas figuras, es posible observar que en ambos casos se observa un muy buen seguimiento de las corrientes de cargas a sus respectivas referencias. También se observa que la ondulación o el error entre las corrientes de salidas obtenidas y las corrientes de referencias disminuye al disminuir el tiempo de muestreo. La Fig. 7 muestra la señal de la corriente de carga obtenida al aplicar la técnica de control MPC a frecuencia de conmutación fija, a un tiempo de muestreo de $T_{s}=25[\mu s]$, se observa que la ondulación de la corriente de carga es ligeramente menor que el método predictivo clásico, para este tiempo de muestreo, eso también se puede observar en la Fig. 7b que tiene un voltaje de carga más sinusoidal con respecto a la tensión de carga de la Fig. 5b.

La naturaleza de la frecuencia de conmutación variable se puede ver claramente con el método de control predictivo clásico, observando la tensión sobre la carga en las Figs. 5b y $\mathbf{6 b}$, mientras que el esquema de control predictivo a frecuencia fija, mantiene constante la frecuencia de conmutación, Fig. 7b, presentando una mejor señal senoidal.

\section{B. Respuesta transitoria}

El análisis transitorio se lleva a cabo con ambos métodos de control aplicado al MMMC. Las Figs. 8a y 8 b muestran
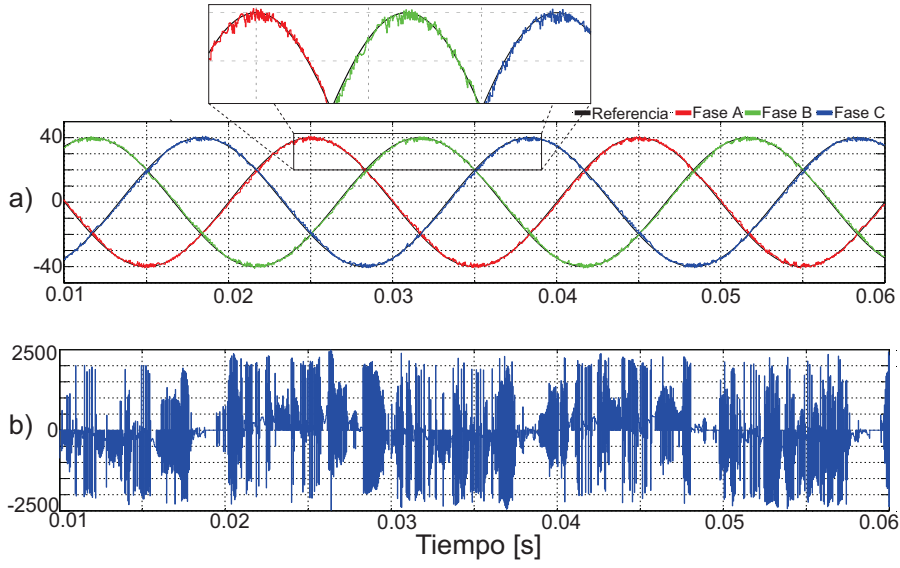

Fig. 5. a) señal de la corriente de carga obtenida al aplicar la técnica de control MPC clásico al SPMC multimodular, a un tiempo de muestreo de $\left.T_{s}=25[\mu s], b\right)$ señal de la tensión de carga obtenida bajo dichas condiciones.
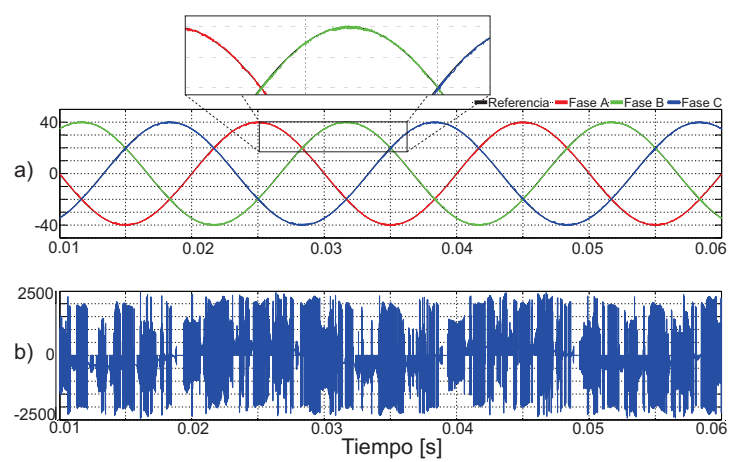

Fig. 6. a) señal de la corriente de carga obtenida al aplicar la técnica de control MPC clásico al SPMC multimodular, a un tiempo de muestreo de $\left.T_{s}=10[\mu s], b\right)$ señal de la tensión de carga obtenida bajo dichas condiciones.

los resultados para el método predictivo clásico y control predictivo a frecuencia de conmutación fija, respectivamente, ambos en condiciones de funcionamiento similares, se aplica un cambio de paso en la referencia de corriente $i_{\text {ref }}$ de $40[A]$ a $20[A]$, en el instante $t=0.03[s]$. En ambos casos se observa muy buena respuesta dinámica. Se observa que la ondulación de la corriente de carga es menor para el esquema de control predictivo a frecuencia de conmutación fija, sin embargo, la respuesta dinámica del control predictivo clásico es mayor que el del control predictivo a frecuencia fija, debido a que este último tiene mayor cálculo que realizar.

\section{Análisis en términos de distorsión armónica total (THD)}

Finalmente, la figura de méritos utilizado en el presente trabajo es la distorsión armónica total de la señal de corriente eléctrica generada en la salida del convertidor de potencia. En las Figs. 9 y 10 se presentan los espectros de frecuencia tanto para el control predictivo a frecuencia variable Fig. 9 como para el control predictivo a frecuencia fija Fig. 10. Para el esquema de frecuencia variable, es posible apreciar mayor densidad espectral a frecuencias bajas, se observa una 

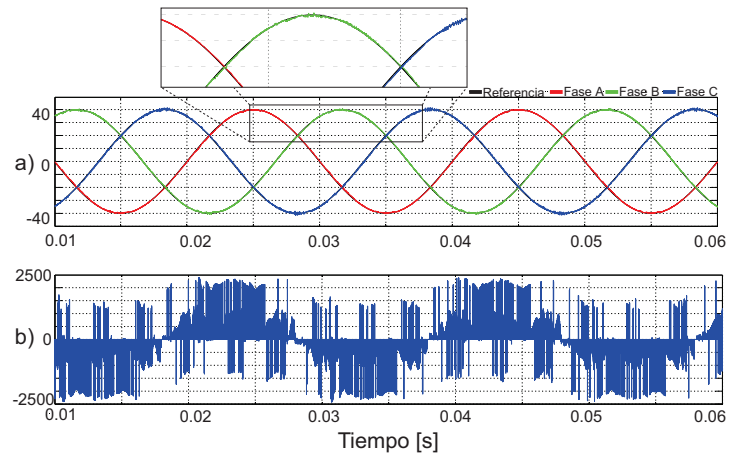

Fig. 7. a) Señal de la corriente de carga obtenida al aplicar la técnica de control MPC a frecuencia de conmutación fija al SPMC multimodular, a un tiempo de muestreo de $\left.T_{s}=25[\mu \mathrm{s}], b\right)$ señal de la tensión de carga obtenida bajo dichas condiciones.

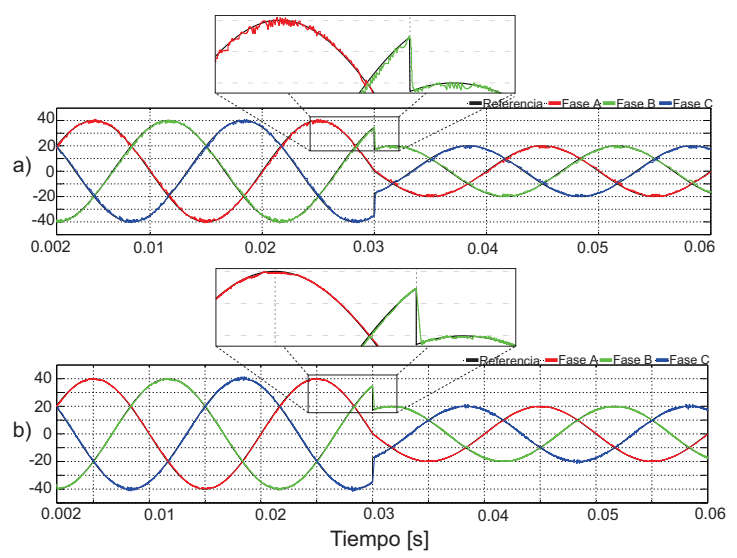

Fig. 8. a) Señal de la corriente de carga obtenida al aplicar la técnica de control MPC tradicional al SPMC multimodular, para una cambio instantáneo de referencia, a un tiempo de muestreo de $\left.T_{s}=25[\mu s], b\right)$ Señal de la corriente de carga obtenida al aplicar la técnica de control MPC a frecuencia de conmutación fija al SPMC multimodular, para un cambio instantáneo de referencia, a un tiempo de muestreo de $T_{s}=25[\mu s]$.

concentración importante entorno a los $10[\mathrm{kHz}]$ para una frecuencia de muestreo de $40[k H z]$. Para el esquema de frecuencia de conmutación fija, es posible observar claramente la componente en la frecuencia de muestreo. Con ello se confirma que el algoritmo logra este objetivo particular, pues los interruptores están conmutando en cada tiempo de muestreo. En cuanto al control predictivo clásico, posee un espectro bastante amplio lo que dificulta el diseño de filtros con frecuencia de corte baja, por su parte, la técnica de control predictivo de conmutación a frecuencia fija logra concentrar el espectro en torno a la frecuencia de muestreo, por lo cual posee menor cantidad de componentes a frecuencias bajas y una clara componente ubicada justamente a la frecuencia de muestreo, que en general, es de valor elevado lo que implica la utilización de dispositivos más veloces, mayor emisión electromagnética y más pérdidas por conmutación.

\section{CONCLUSIÓN}

La estrategia de control propuesta presenta un correcto desempeño, tanto desde el punto de vista dinámico, pues

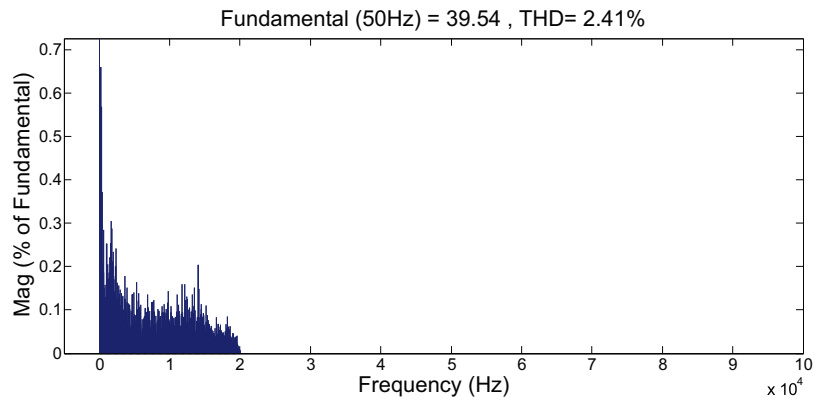

Fig. 9. THD de la corriente de carga de la técnica de control MPC a frecuencia variable aplicado al SPMC multimodular.

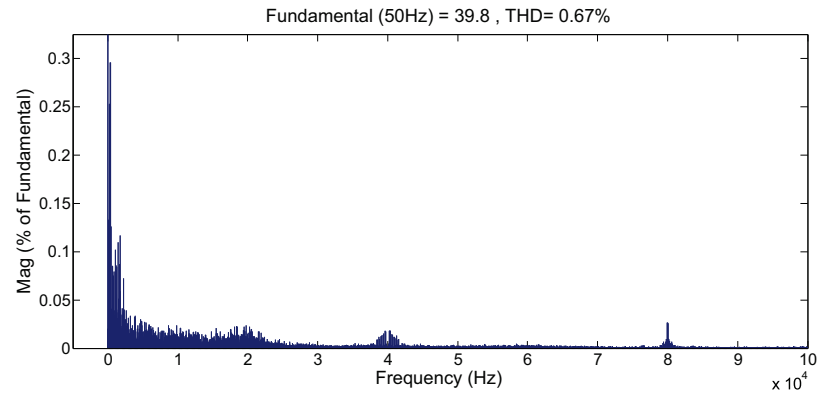

Fig. 10. THD de la corriente de carga de la técnica de control MPC a frecuencia fija aplicado al SPMC multimodular.

los transitorios son suficientemente cortos y en estado estacionario, pues el seguimiento es logrado en dicho estado. En cuanto a la respuesta armónica, el THD cumple ampliamente con los requerimientos de los estándares internacionales y se puede mencionar que el espectro de armónicos se encuentra mejor distribuido, es decir, en frecuencias múltiplos a la de la conmutación de los semiconductores, se tienen picos de armónicos que pueden ser eliminados con filtros pasivos, ya que se conocen estas frecuencias. En este enfoque la estrategia de control a frecuencia de conmutación fija mantiene las ventajas del MPC tradicional como respuesta dinámica rápida, control multiobjetivo, fácil inclusión de no linealidades y restricciones, pero agrega una operación que permite reducir las ondulaciones de las variables controladas y mejorar el desempeño general del sistema. Se aplicó con éxito el control MPC aplicado al convertidor matricial multi-modular que hoy en día es un tema muy importante debido a la creciente necesidad de convertidores de potencia de alto voltaje para diversas aplicaciones. Estos resultados obtenidos en las simulaciones demuestran que esta técnica de control MPC a frecuencia de conmutación fija, es una alternativa viable para el control de convertidores matriciales multi-modular, que funciona bien en condiciones constantes y transitorias, con un buen seguimiento de sus referencias y una ondulación reducida.

\section{AgRAdeCIMIENTOS}

Los autores agradecen el apoyo económico del Proyecto de Investigación FONDECYT Regular 1191028, MEC 80190074, MEC 801800974 y FONDAP SERC Chile 15110019. 


\section{REFERENCIAS}

[1] S. Toledo, M. Ayala, E. Maqueda, R. Gregor, A. Renault, M. Rivera, T. Dragicevic, and P. Wheeler, "Active and reactive power control based on predictive voltage control in a six-phase generation system using modular matrix converters," in 2020 IEEE International Conference on Industrial Technology (ICIT), 2020, pp. 1059-1065.

[2] M. Rivera, G. Castro, and P. Wheeler, "Design, assembly and startup of a single-phase multi-modular matrix converter for grid interconnection," in 2018 IEEE International Conference on Automation/XXIII Congress of the Chilean Association of Automatic Control (ICA-ACCA), 2018, pp. $1-6$.

[3] M. Majstorović, M. E. R. Abarca, and L. Ristic, "Review of mpc techniques for mmcs," in 2019 20th International Symposium on Power Electronics (Ee), 2019, pp. 1-7.

[4] V. Yaramasu, K. Milev, A. Dekka, M. Rivera, J. Rodriguez, and F. Rojas, "Modulated model predictive current control of a four-leg inverter,' in 2020 11th Power Electronics, Drive Systems, and Technologies Conference (PEDSTC), 2020, pp. 1-6.

[5] S. Toledo, E. Maqueda, M. Rivera, T. Dragicevic, and R. Gregor, "Predictive voltage control techniques for ac ups applications," in 2018 IEEE International Conference on Automation/XXIII Congress of the Chilean Association of Automatic Control (ICA-ACCA). IEEE, 2018, pp. 1-6.

[6] M. Rivera, S. Toledo, L. Tarisciotti, P. W. Wheeler, and H. Dan, "Predictive control strategies operating at fixed switching frequency for input filter resonance mitigation in an indirect matrix converter," IEEE Latin America Transactions, vol. 16, no. 9, pp. 2370-2376, 2018.

[7] P. Blaszczyk, "Hex-y — a new modular multilevel converter topology for a direct ac-ac power conversion," in 2018 20th European Conference on Power Electronics and Applications (EPE'18 ECCE Europe), 2018, pp. P.1-P.10.

[8] M. Majstorović, M. E. R. Abarca, and L. Ristic, "Review of mpc techniques for mmcs," in 2019 20th International Symposium on Power Electronics (Ee), 2019, pp. 1-7.

[9] W. Xiong, Y. Sun, M. Su, J. Yang, and C. Wang, "A carrier-based modulation strategy for multi-modular matrix converters with zero common-mode voltage," in 2016 IEEE Energy Conversion Congress and Exposition (ECCE), 2016, pp. 1-6.

[10] S. Rojas, M. Rivera, and P. Wheeler, "Control techniques for a singlephase matrix converter," in 2019 IEEE CHILEAN Conference on Electrical, Electronics Engineering, Information and Communication Technologies (CHILECON), 2019, pp. 1-7.

[11] Y. Luo and C. Liu, "Multi-vector-based model predictive torque control for a six-phase pmsm motor with fixed switching frequency," IEEE Transactions on Energy Conversion, vol. 34, no. 3, pp. 1369-1379, 2019.

[12] Q. Wang, H. Yu, C. Li, X. Lang, S. S. Yeoh, T. Yang, M. Rivera, S. Bozhko, and P. Wheeler, "A low-complexity optimal switching timemodulated model-predictive control for pmsm with three-level $\mathrm{npc}$ converter," IEEE Transactions on Transportation Electrification, vol. 6, no. 3, pp. 1188-1198, 2020. 